\title{
Probiotics Can Break the Toxic Relationship Between the Intestinal Microbiome and the Kidney
}

\author{
Luis Vitetta ${ }^{1}$ \\ Published online: 2 November 2018 \\ (c) Springer Science+Business Media, LLC, part of Springer Nature 2018
}

The human microbiome project [1] has systematically advanced knowledge of how the bacteria that cohabitate with humans interact with the host, creating opportunities to improve health from the study of the beneficial health effects of the human microbiome. A renewed interest in an old posit stems from the idea that the colonic complement of commensal and pathogenic bacteria can be "encouraged" to improve health outcomes as a consequence of the inclusion of "beneficial" bacterial species and fermentable carbohydrates (fiber or prebiotics) in the diet. Recent interest in microbiome research in the field of nephrology has been reinvigorated by the application of molecular methods to profile and characterize the human microbiome, combined with the realization that the intestinal microbiome has wideranging extraintestinal effects, including on the kidney [1]. The intestinal microbiome, reported to exhibit the most complex and diverse taxonomic profile [2], has been cited as the principal contributors to the progression of kidney disease, associated with shifts in its taxa [3]. Numerous factors can modify the intestinal microbiota such as antibiotics, dietary alteration, and intestinal infection with pathogens [4]. Such disruption to the composition of the intestinal microbiome can lead to dysbiosis, defined as a disproportion in the taxonomic profile of the intestinal bacterial microbiome, associated with an enhanced risk of the development and acceleration of chronic diseases [5].

Local and systemic inflammation supported by the overproduction of uremic toxins follows a dysbiotic bacterial cohort that mostly occupies the colonic lumen [6]. In patients with advanced chronic kidney disease (CKD), uremic toxins (e.g., $p$-cresyl and indoxyl sulfates and bloodbound proteins poorly cleared by dialysis) are consistently

Luis Vitetta

luis.vitetta@sydney.edu.au; luis_vitetta@medlab.co

1 Sydney Medical School, Faculty of Medicine and Health, The University of Sydney and Medlab Clinical, Sydney, NSW, Australia associated with increased severity of CKD [3]. Uremic toxins are hypothesized to exert harmful effects via augmentation of glucotoxicity and lipotoxicity, increased activity of the hexosamine and polyol biosynthetic pathways, and the accumulation of advanced glycation end products that are associated with a uremic dysbiotic gut [7]. The colonic environment is subsequently altered through the active accumulation of numerous endogenous microbial metabolites that include uric acid, urea, and oxalate. These gut-derived uremic toxin metabolites that are normally excreted by the kidneys are transported across the intestinal epithelium and into the systemic circulation where they are retained [3].

Probiotics are lactic acid-producing bacteria with an extensive documented history of beneficial effects relative to their presence in fermented dairy products, originating from records that predate antiquity [8]. A consensus panel convened by the International Scientific Association of Probiotics and Prebiotics defined probiotics as live microorganisms that, when administered in sufficient quantities, confer a health benefit to the host [9].

The gradual loss of kidney function and progression to the advanced stage of CKD is termed as end-stage renal disease (ESRD). In this issue of Digestive Diseases and Sciences, a systematic review and meta-analysis conducted by Thongprayoon et al. [10] demonstrated that the administration of probiotics provides a potential health benefit in patients maintained on dialysis for ESRD. Study selection for inclusion in the meta-analysis followed a systematic literature search that identified clinical studies that assessed the efficacy of probiotics versus a control comparator (usually a placebo when reported). Thongprayoon et al. [10] reported the combined analyses of seven clinical studies incorporating 178 ESRD patients designed to evaluate the effects of probiotics on inflammatory markers, uremic toxin retention solutes, and gastrointestinal symptomatology. The systematic review and meta-analysis concluded that there was a significant reduction in the standardized mean difference (i.e., a measure of effect size in favor of 
probiotics over the control comparator) for the systemic inflammatory marker C-reactive protein, protein-bound uremic toxins, and overall improvement in gastrointestinal symptoms in those patients provided with a probioticcontaining formula. Their meta-analysis provides a useful justification for the administration of probiotics for the efficacious treatment of patients with ESRD maintained on chronic hemodialysis.

As Thongprayoon et al. [10] have discussed, there was an overall low-level significant trend in heterogeneity of the clinical studies included in the meta-analysis. Moreover, the authors also considered treatment duration and follow-up times that were often too brief and varied to be able to detect significant changes in the positive inflammatory biomarker serum tumor necrosis factor (TNF)- $\alpha$ or the inverse inflammatory marker albumin, markers associated with ESRD severity and mortality risk. Given that it was highly likely that the clinical studies chosen displayed interstudy variance, a random effect model was chosen in order to determine the interstudy heterogeneity. This variance model assumed no fixed effects for the ESRD population and the probiotic formulations investigated from the seven clinical studies, an acceptable statistical model given their heterogeneity. As a consequence, the meta-analysis reported statistically significant efficacy for a wide variety of probiotic species in ESRD patients maintained on dialysis. Furthermore, since dialysis patients have low-fiber intake, this could also contribute to dysbiosis [11]. Since dietary fiber, which is comprised of fermentable carbohydrates, is the most recognized modifier of the intestinal microbiome composition [12], their inclusion in the formulations of probiotics used in four of the clinical studies certainly warrants further attention.

Results from systematic reviews reporting the effectiveness of administration of diverse probiotic formulations can bias the mechanistic understanding of how probiotics affect the host. More importantly, reporting efficacy outcomes from heterogeneous clinical studies that use dissimilar probiotic formulations with single or multiple species in the presence of prebiotics can skew efficacy interpretations that may not be translatable to other clinical states. Such wide-ranging efficacy of probiotics derived from meta-analyses can often be used by probiotic manufacturers to make unreasonable and scientifically unsupported claims about the efficacy of their products in lieu of researching single probiotic formulations using large controlled clinical trials.

The mechanisms by which probiotics exert beneficial effects on the intestines may include strengthening of the intestinal epithelial barrier, promoting and sustaining local immunological homeostasis, inducing favorable changes in intestinal luminal $\mathrm{pH}$, and suppressing the activity of pathobionts (pathogenic intestinal bacteria) through competitive exclusion and antagonism for available nutrients [13]. The overall effect is to encourage the intestinal microbiome to maintain a favorable proinflammatory/anti-inflammatory balance with the host.

This study, irrespective of the composition of the probiotic formulation used, has convincingly shown that certain probiotics are associated with a reduction in the gut burden of a wide range of uremic toxins that in turn can decrease concentrations of retained circulating uremic solutes. It is possible that probiotics combined with prebiotics as synbiotic formulations can shift the intestinal microbiome toward a composition associated with an increased production of the bacterial metabolites termed short-chain fatty acid such as butyrate [14] that can help reduce the burden of circulating retained protein-bound uremic solutes in chronic kidney diseases.

Although probiotics are not a panacea, they are relatively inexpensive and nontoxic. This systematic review and metaanalysis has advanced a plausible premise for the administration of probiotics to patients with CKD. Robust studies with longer treatment duration with formulations consisting of multiple probiotic species with additional fiber are certainly warranted.

\section{Compliance with ethical standards}

Conflict of interest Luis Vitetta has received National Institute of Complementary Medicine and National Health and Medical Research Council of Australia competitive funding and Industry support for research into probiotics and the gut microbiome. The author has no further conflicts of interest relevant to the content of this editorial.

\section{References}

1. Peterson J, Garges S, Giovanni M, et al. The NIH human microbiome project. Genome Res. 2009;19:2317-2323.

2. Consortium ThMP. Structure, function and diversity of the healthy human microbiome. Nature. 2012;486:207-214.

3. Lau WL, Savoj J, Nakata MB, Vaziri ND. Altered microbiome in chronic kidney disease: systemic effects of gut-derived uremic toxins. Clin Sci (London, England: 1979). 2018;132:509-522.

4. Karczewski J, Poniedzialek B, Adamski Z, Rzymski P. The effects of the microbiota on the host immune system. Autoimmunity. 2014;47:494-504.

5. Yang T, Richards EM, Pepine CJ, Raizada MK. The gut microbiota and the brain-gut-kidney axis in hypertension and chronic kidney disease. Nat Rev Nephrol. 2018;14:442-456.

6. Yang CY, Tarng DC. Diet, gut microbiome and indoxyl sulphate in chronic kidney disease patients. Nephrology (Carlton, VIC). 2018;23:16-20.

7. Koppe L, Mafra D, Fouque D. Probiotics and chronic kidney disease. Kidney Int. 2015;88:958-966.

8. Ozen M, Dinleyici EC. The history of probiotics: the untold story. Benef Microbes. 2015;6:159-165.

9. Hill C, Guarner F, Reid G, et al. Expert consensus document. The international scientific association for probiotics and prebiotics consensus statement on the scope and appropriate use of the term probiotic. Nat Rev Gastroenterol Hepatol. 2014;11:506-514. 
10. Thongprayoon C, Kaewput W, Hatch ST, et al. Effects of probiotics on inflammation and uremic toxins among patients on dialysis: a systematic review and meta-analysis. Dig Dis Sci. (Epub ahead of print). https://doi.org/10.1007/s10620-018-5243-9.

11. Sirich TL, Plummer NS, Gardner CD, Hostetter TH, Meyer TW. Effect of increasing dietary fiber on plasma levels of colonderived solutes in hemodialysis patients. Clin J Am Soc Nephrol CJASN. 2014;9:1603-1610.

12. Sonnenburg JL, Backhed F. Diet-microbiota interactions as moderators of human metabolism. Nature. 2016;535:56-64.
13. Vitetta L, Linnane AW, Gobe GC. From the gastrointestinal tract (GIT) to the kidneys: live bacterial cultures (probiotics) mediating reductions of uremic toxin levels via free radical signaling. Toxins. 2013;5:2042-2057.

14. Chen J, Vitetta L. Inflammation-modulating effect of butyrate in the prevention of colon cancer by dietary fiber. Clin Colorectal Cancer. 2018;17:e541-e544. 